\title{
Herschel spectroscopic observations of PPNe and $\mathrm{PNe}$
}

\author{
Pedro García-Lario ${ }^{1}$, J. Ramos-Medina ${ }^{2}$ and C. Sánchez-Contreras ${ }^{2}$ \\ ${ }^{1}$ European Space Astronomy Centre, ESA, E-28691 Villanueva de la Cañada, Madrid, Spain \\ email: pedro.garcia.lario@esa.int \\ ${ }^{2}$ Centro de Astrobiología (CSIC-INTA), E-28691 Villanueva de la Cañada, Madrid, Spain
}

\begin{abstract}
We are building a catalogue of interactively reprocessed observations of evolved stars observed with Herschel. The catalogue will offer not only the PACS and SPIRE spectroscopic data for each observation, but also complementary information from other infrared space observatories. As a first step, we are concentrating our efforts on two main activities: 1) the interactive data-reduction of more than 500 individual spectra obtained with PACS in the 55-210 $\mu \mathrm{m}$ range, available in the Herschel Science Archive; 2) the creation of a catalogue, accesible via a web-based interface and through the Virtual Observatory. Our ultimate goal is to carry out a comprehensive and systematic study of the far infrared properties of low-and intermediate-mass evolved stars using these data and enable science based on Herschel archival data. The objects cover the whole range of possible evolutionary stages in this short-lived phase of stellar evolution, from the AGB to the PN stage, displaying a wide variety of chemical and physical properties.
\end{abstract}

\section{Introduction}

More than 200 evolved stars were observed in spectroscopic mode with Herschel (Pilbratt et al. 2010) either with PACS $(55-210 \mu \mathrm{m})$ or SPIRE $(200-670 \mu \mathrm{m}) .71 \%$ of them correspond to low-or intermediate-mass stars, $28 \%$ are massive stars $\left(>8 \mathrm{M}_{\odot}\right)$ and the $1 \%$ left are objects that are difficult to classify. Our work is initially focused on the lowand intermediate-mass evolved stars subsample for which PACS spectroscopic data is available and covers the whole range of evolutionary stages: from AGB stars (39\%) to PNe (22\%), including the rapidly evolving post-AGB (25\%) and OH/IR stars (14\%).

The automated standard data reduction pipelines used for the generation of PACS data products lack some important corrections that require in many cases an interactive analysis: spectral flat-fielding, telescope background correction, and other data reduction tasks which take into account the actual fraction of the PSF that falls out of the PACS central 'spaxel' or the slight mispointings affecting some observations are examples of the kind of corrections that can significantly improve the $\mathrm{S} / \mathrm{N}$ ratio of the PACS spectra and, more important, the absolute flux calibration.

\section{The THROES on-line catalogue}

THROES is an on-line queriable catalogue which contains in this first release the interactively reduced PACS spectra for all evolved stars observed with Herschel. In future releases it will be further populated with the result of the interactive data reduction of the corresponding SPIRE spectroscopy observations, something on which we are currently working. For each catalogue entry, a number of columns are provided with information about the observation (Coordinates, Target Name or Observing Mode/AOT), as well as 


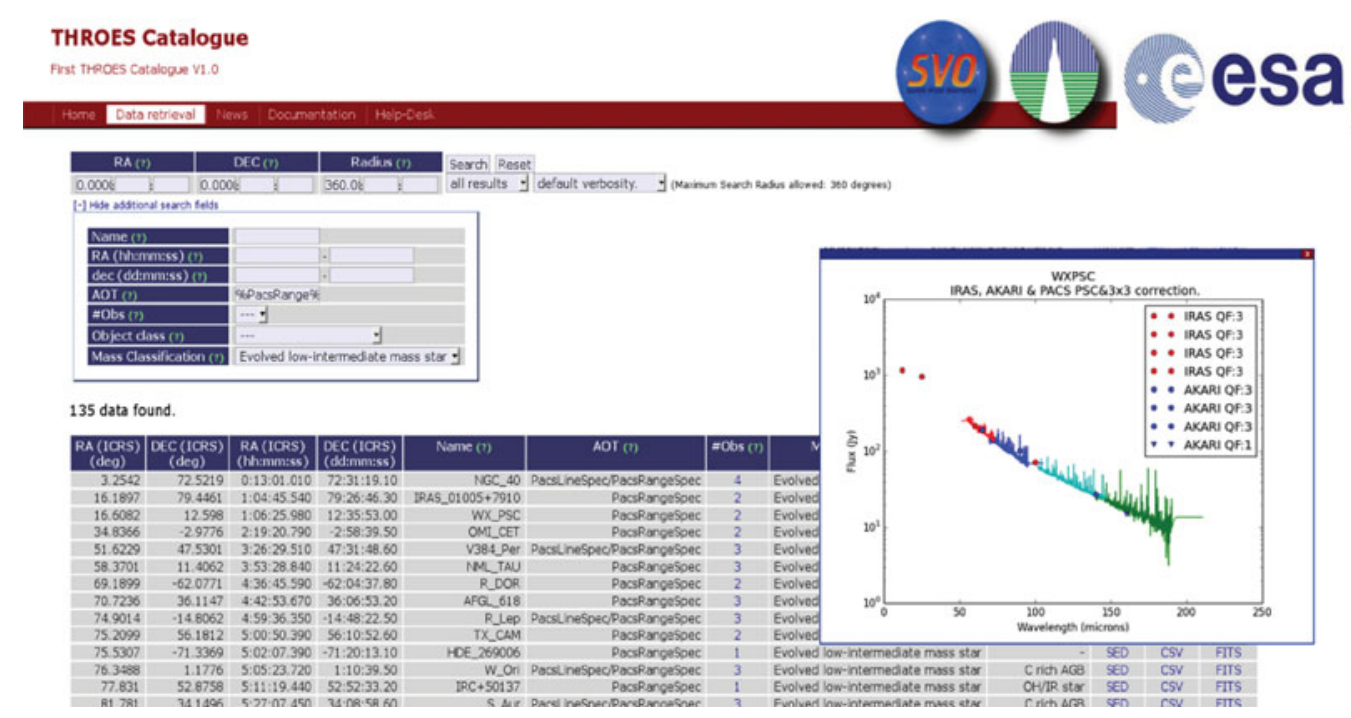

Figure 1. The THROES on-line catalogue web-based user interface.

on the object itself (low-intermediate or high mass, chemical composition - C-rich/Orich - and evolutionary stage), including the interactively reduced PACS data, that can be downloaded in various formats or displayed for quick look, together with IRAS and AKARI photometric data (Fig. 1).

The ultimate goal of this project is enabling science exploitation of Herschel archival data. With its improved spatial resolution compared to previous space facilities covering the same spectral range (e.g LWS onboard ISO), larger FoV and better sensitivity, Herschel has the potential to lead us to a better understanding of the physical and chemical evolution experienced by evolved stars at the end of their life. Science analysis based on Herschel/PACS includes the determination of the physical and chemical properties of the molecular gas in the inner regions of their circumstellar envelopes through the analysis of the wealth of rotational molecular lines $\left(\mathrm{CO}, \mathrm{OH}, \mathrm{H}_{2} \mathrm{O}\right.$, etc) displayed by many of the stars in the sample, or the analysis of dust species like forsterite $\left(\mathrm{Mg}_{2} \mathrm{SiO}_{4}\right)$ at $69 \mu \mathrm{m}$ or calcite $\left(\mathrm{CaCO}_{3}\right)$ at $92.6 \mu \mathrm{m}$ that may be contained in the coolest regions of some of the envelopes. Additionally, in proto-PNe and PNe, atomic/ionized nebular emission lines are sometimes detected and can also be studied.

\section{Reference}

Pilbratt, G. L., Riedinger J. R., Passvogel T., et al. 2010, A\&A, 518, L1 